\title{
26410 - THE 'ANALGOSCORE': A NEW SCORE FOR INTRAOPERATIVE PAIN USED FOR FEEDBACK ADMINISTRATION OF REMIFENTANIL
}

\section{Emile Salhab MSc, Stephane Deschamps, MSc; Pierre Mathieu, PhD; Thomas Hemmerling, University Of Montreal, Montreal, PQ, Canada}

Objective. The focus of this project is the development of a novel score (called: Analgoscore) of intraoperative pain based on fuzzy-logic algorithms using heart rate (HR) and blood pressure (BP). The 'Analgoscore' was integrated in a closed-loop application of remifentanil.

METHODS. A score (and PC-based interface) was developed ranging from -9 to 9 in increments of 1 , with $0 \pm 3$ reflecting excellent pain control, -3 to -6 and 3 to 6 good pain control and -6 to -9 and 6 to 9 insufficient pain control, respectively with positive score values representing insufficient analgesia and negative score values unnecessarily profound analgesia. (Figure 3). According to the zone of analgesia control (excellent, good, insufficient), a remifentanil infusion was closed-loop-administered according to predefined algorithms. In this pilot study, 10 patients undergoing surgery of moderate trauma were included. The 'Analgoscore' was determined every 1 min, BP noninvasively and HR with pulse oxymetry. The percentage of time within the different score zones was recorded and the times when artifacts occurred.

RESULTS. Ten patients, ( $5 \mathrm{f}, 5 \mathrm{~m} ; 52 \pm 22$ y) underwent 1 MAC-sevoflurane -anesthesia of 98 (31) min, with a mean dose of remifentanil of $0.14 \pm 0.01 \mathrm{ig} / \mathrm{kg} / \mathrm{min}$. The Figure 1 shows controller performance with $80 \%$ of the time excellent control. During only $1 \%$ of the time, artifacts were recorded.

DISCUSSION. We present the 'Analgoscore' to measure intraoperative pain; good and excellent control is related with good hemodynamic stability. The 'Analgoscore' successfully drove a closed loop administration of remifentanil. Future studies should compare our system with manual administration of remifentanil and compare hemodynamic stability.

Figure 1 a: Controller performance for 10 patients

Figure 1 b: User Interface
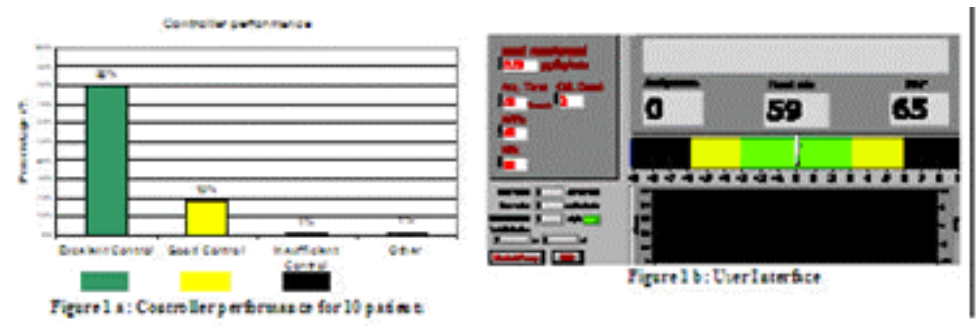